\title{
Acoustic-electric conversion and piezoelectric properties of electrospun polyvinylidene fluoride/silver nanofibrous membranes
}

\author{
C. M. Wu ${ }^{*}, M . H$. Chou
}

Department of Materials Science and Engineering, National Taiwan University of Science and Technology, 10607 Taipei, Taiwan, R.O.C.

Received 4 May 2019; accepted in revised form 23 July 2019

\begin{abstract}
Herein, a novel sound energy acquisition device based on a flexible electrospun polyvinylidene fluoride (PVDF) nanofibrous membrane was developed. Effects of electrospinning and the addition of silver nanoparticles (AgNPs) on the crystal structure and piezoelectric properties of the PVDF nanofibrous membranes were examined. Electrospinning and the addition of AgNPs effectively induced the formation of the $\beta$-phase and increased the piezoelectricity. The piezoelectric electrospun PVDF samples were crucial for converting sound energy into electric potential and absorbing sound waves. Moreover, the use of piezoelectric shunt damping reduced the sound transmission at low frequencies. Therefore, the electric energy generated in the low-frequency region was higher than in mid- and high-frequency regions. The electric power of PVDF/AgNP was $7 \cdot 10^{-4} \mathrm{~W}$ - a significant increase of $40 \%$ compared to PVDF without AgNPs $\left(5 \cdot 10^{-4} \mathrm{~W}\right)$ at low frequency. This demonstrates that PVDF/AgNP presents excellent piezoelectric properties and acoustic-electric conversion characteristics. Hence, the piezoelectric PVDF device fabricated herein can not only reduce sound transmission but also convert the sound energy into electric energy. This novel sound energy acquisition device is practical and efficient in various sensing and energy harvesting applications, and will aid in the operation of low-power consumer electronic devices and to maintain a green environment.
\end{abstract}

Keywords: nanocomposites, electrospinning, polyvinylidene fluoride, silver, acoustic-electric conversion

\section{Introduction}

Research on energy harvesting technologies has been actively carried out owing to the advancements in low-power wireless sensor technologies [1]. Energy can be harvested from different sources such as mechanical vibration and electromagnetic, light, wind, and temperature variations. Energy is freely obtainable from the environment to complement the lowpower sources used for energizing low-power electronic devices; this constitutes 'green technology'. Among the various energy sources, the sound energy generated from the surroundings can be harvested and widely utilized. The main advantages of sound wave-based energy harvesting systems are the minimum maintenance required and the feasibility of large-scale implementation in previously inaccessible locations. Recent studies have focused on transforming the ambient energy into practical and usable electrical energy via transduction mechanisms such as electromagnetic, electrostatic, or piezoelectric transduction [2, 3]. Piezoelectric materials are used for harvesting energy from ambient vibration sources because they can efficiently convert mechanical strain to electric charges without additional power.

Polymeric piezoelectric materials such as polyvinylidene fluoride (PVDF) are among the widely 
investigated piezoelectric materials because of their good piezoelectricity and conformable properties, which enable their application to complex structural surfaces. These materials are light, strong, and conformable, and can be cut into various shapes and sizes. Furthermore, the polymeric nature and chemical compatibility of PVDF allow precise tailoring of the material using other nanomaterial species. PVDF is a semi-crystalline polymer with five crystalline phases and three conformations [4]: (1) the all-trans (TTTT) planar zigzag conformation for the $\beta$-phase, (2) TGTG' for the $\alpha$ - and $\delta$-phases, and (3) T3GT3G' for the $\gamma$ - and $\varepsilon$-phases. The $\alpha$-phase is nonpolar and is typically found in commercial PVDF films, whereas the $\beta$-phase is polar. In the $\beta$-phase, dipole moments are oriented in the same direction; therefore, this phase determines the piezoelectric properties of PVDF polymers [5]. To obtain a highly piezoelectric PVDF, formation of the polar $\beta$-phase is vital. Mechanical stretching [6] and high-electric-field poling [7-10] are critical for the immobilization of the polar phase. Recent approaches have aimed at obtaining the $\beta$-phase of PVDF through electrospinning and incorporating PVDF with nanoparticles [11-13].

Electrospinning is a manufacturing technique which can be used to extract continuous nanofibers with several favorable characteristics such as a low diameter, high specific surface area, flexibility in surface functionality, and high porosity [14-16]. PVDF nanofibrous membranes have been successfully prepared via electrospinning, and their $\beta$-phase content has been significantly enhanced [17-19]. Furthermore, electrospinning confines the polymer in fibers of small diameters, which favors $\beta$-phase formation. This action may be enhanced by the presence of nanofillers, because during spinning of a polymer, the chains are forced to elongate as fibers of small diameters, while overcoming the resistance to flow produced by the nanofillers [20-22].

Silver is a material that attracts the interest of many researchers on account of their high aspect ratio, and good thermal and electrical conductivities [23]. Wu added silver nanowires (AgNWs) to poly(vinylidene fluoride-co-hexafluoropropylene) (PVDF-HFP) to prepare composites with excellent piezoelectricity [24], and proved the positive effect of the moderate addition of AgNWs on the formation of the $\beta$-phase and phase transformation. Li increased the crystalline $\beta$-phase content in PVDF by adding AgNWs [25]. The crystalline $\beta$-phase fraction could reach up to $55 \%$ when doped with $1.5 \mathrm{wt} \% \mathrm{AgNWs}$ in PVDF nanofiber webs. Besides, silver nanoparticles (AgNPs) also have been predominantly used as ideal fillers in polymer matrixes as they can greatly enhance the crystal $\beta$-phase structure of PVDF by Issa's group [26]. They pointed that adding AgNPs in PVDF can obtain higher $\beta$-phase content, approximately $80 \%$. Electron-rich AgNPs interacts with fluorine atoms in PVDF for enhancing polarization, thus increasing the $\beta$-phase content. Therefore, the presence of electrically conductive particles in nanofibers during electrospinning process can enhance polarization.

Furthermore, noise control is vital and has attracted attention from governments and academia for increasing the quality of life. Passive and active noise mitigation are two general approaches for reducing noise. Passive noise mitigation uses noise-isolating or sound-absorbing materials and is easy to implement without complicated control algorithms or energy consumption as required in the active method. Commercial sound-absorbing materials include mineral wool, plastic foams, textiles, cotton, and special acoustic plasters. The efficiency of these absorbers is often insufficient, especially in the low-frequency region below $2 \mathrm{kHz}$; improving this efficiency has always been challenging because the dissipation of materials is inherently weak in this regime [27, 28]. Therefore, to improve the low- and mid-frequency absorption, other mechanisms are required.

Some research groups found that piezoelectric shunt damping technology can reduce sound transmission [29-31]. They investigated this effect to determine the mechanism for improving the sound transmission loss of a smart plate with shunt circuits. Lang's group also pointed piezoelectric materials can precisely distinguish sound waves in low- to middlefrequency region. These features make them especially suitable for noise detection [32]. Furthermore, we have also previously reported that the piezoelectric electrospun PVDF plays a crucial role in absorbing sound at the low-frequency region $[18,19]$. Meanwhile, electrospun nanofibrous structures would increase the sound-absorbing coefficient to more than that of conventional materials such as nonwoven and acoustic foams because of their high surface area, porosity, and flexibility. Moreover, the frequency of the nanofibers decreases with increasing areal density of the nanofibrous membranes, which enhances the mid-frequency absorption. Therefore, piezoelectric 
materials can not only be used to reduce vibrations and noise, but can also be employed in electric energy acquisition applications. Determining efficient ways to harvest the environmental noise into electrical energy is challenging as the acoustic energy originates from the low power density. Recently, some efficient piezoelectric energy harvesting interface circuits have been developed; these are typically used to extract power from piezoelectric energy harvesters.

Thus far, for harvesting sound energy, researchers have mainly focused on acoustic sensor applications. These sensors can detect the defects and cracks in a structure by capturing the acoustic-electric transformation generated owing to crack opening and propagation [33-35]. However, the piezoelectric effect has not been successfully explored as a passive approach for acoustic control, especially in terms of its electric output at different acoustic frequencies. Piezoelectric PVDF nanofibrous membranes can reduce sound transmission and further convert sound energy to various other energies, including electric current. Therefore, in this study, AgNPs were added and co-electrospun with PVDF to enhance the piezoelectric properties of the membranes. A novel sound energy acquisition device was developed. The piezoelectricity, sound-absorbing property, and acousticelectric conversion of the PVDF/AgNP nanofibrous membranes were investigated.

\section{Experimental section}

\subsection{Materials}

PVDF pellets $\left(M_{\mathrm{W}}=263000 ; \operatorname{Kynar}^{\circledR} 720\right.$, Arkema Group, France) and AgNP/DMF (20000 ppm; Liho Chem. Co., Taiwan) were used in this study. Indium tin oxide-coated polyethylene terephthalate (ITO/ PET; Sigma-Aldrich, Taiwan) with a sheet resistivity of $200 \Omega$ /square and a thickness of $0.13 \mathrm{~mm}$ was used as the substrate for electrospinning. Acoustic foam (BSWA Technology Co., China), containing polyurethane (PU) and of thickness $150 \mathrm{~mm}$, was used as the acoustic substrate.

\subsection{Preparation of electrospun PVDF/AgNP nanofibrous membranes}

PVDF solution was prepared by adding $2.2 \mathrm{~g}$ of PVDF pellets to $6 \mathrm{~mL}$ of DMF solution and stirring gently at approximately $100^{\circ} \mathrm{C}$ for $2 \mathrm{~h}$. The AgNP/ DMF solution $(20000 \mathrm{ppm})$ was sonicated for 30 min using an ultrasonic cleaner, following which the suspended AgNP/DMF solution was crushed for $20 \mathrm{~min}$ at $40 \mathrm{kHz}$ using an ultrasonic cell disruptor. Subsequently, the resultant solution was added to the as-prepared PVDF solution at $100^{\circ} \mathrm{C}$ under stirring and then cooled to room temperature. The PVDF/ AgNP solution (18 wt $\%$ ) used for electrospinning was prepared by adding $4 \mathrm{ml}$ of acetone to the as-prepared PVDF/AgNP solution to enhance evaporation.

An in-house-designed electrospinning device consisting of an injection spinneret was powered by a syringe pump (KDS 101 Series, KD Scientific, USA), connected to a Teflon tube attached to a stainless steel needle (diameter, $0.23 \mathrm{~mm}$ ). A grounded electrostatic controller (SM 4030-24NIR, You-Shang Technical Corp., Taiwan) was connected to the spinneret and collector. The PVDF/AgNP nanofibrous membranes were directly electrospun and deposited onto the ITO/PET substrate under the following electrospinning conditions: applied voltage, $20 \mathrm{kV}$; working distance, $200 \mathrm{~mm}$; and flow rate, $0.05 \mathrm{ml} / \mathrm{h}$. The tensile properties of electrospun PVDF samples were shown in Figure S1. The tensile strength of PVDF/AgNP was higher than PVDF. In addition, $\mathrm{Ni}-\mathrm{Cu}$-coated nonwoven PET (Shin-Yeh Co. Ltd., Taiwan) was used as the electrode; its sheet resistivity and thickness were $0.6 \Omega$ /square and $0.13 \mathrm{~mm}$, respectively.

\subsection{Characterization}

The morphologies of the electrospun PVDF/AgNP nanofibrous membranes were analyzed by scanning electron microscopy (SEM; JSM-6390, Japan, operated at $20 \mathrm{kV}$ ). The membranes prepared by electrospinning PVDF/AgNP directly onto a copper grid coated with a holey carbon film were examined by transmission electron microscopy (TEM; F20, Philips, USA, operated at $200 \mathrm{kV}$ ).

Differential scanning calorimetry (DSC; DSC-4000, Perkin Elmer, USA) analysis of the electrospun PVDF/AgNP nanofibrous membranes was carried out by scanning approximately $10 \mathrm{mg}$ of the membranes from 25 to $230{ }^{\circ} \mathrm{C}$ at a rate of $20^{\circ} \mathrm{C} / \mathrm{min}$ and then maintaining the membranes under isothermal conditions for $3 \mathrm{~min}$ at $230^{\circ} \mathrm{C}$. The membranes were subsequently cooled to ambient temperature at a rate of $20^{\circ} \mathrm{C} / \mathrm{min}$. Here, the nominal melting temperature $\left(T_{\mathrm{m}}\right)$ was defined as the peak of the melting endotherm during the first heating from 25 to $230^{\circ} \mathrm{C}$, and the nominal crystallization temperature $\left(T_{\mathrm{c}}\right)$ was defined as the peak of the crystallization exotherm during cooling from 230 to $25^{\circ} \mathrm{C}$. 
Fourier transform infrared (FTIR) spectroscopy (FTS1000 , Digilab, USA) was performed in the range of $1500-650 \mathrm{~cm}^{-1}$ at a resolution of $4 \mathrm{~cm}^{-1}$. For the FTIR characterization, specimens of dimensions $10 \mathrm{~mm} \times 10 \mathrm{~mm}$ were prepared.

X-ray photoelectron spectroscopy (XPS; VG ESCA Scientific Theta Probe, UK) was performed to characterize the elements of the electrospun $\mathrm{PVDF} / \mathrm{AgNP}$ nanofibrous membranes, using a monochromatic $\mathrm{Al}$ $\mathrm{K} \alpha \mathrm{X}$-ray source at a constant pass energy of $50 \mathrm{eV}$. The pressure in the analysis chamber was maintained at 8-10 Torr, and specimens of dimensions $2 \mathrm{~mm} \times$ $2 \mathrm{~mm}$ were prepared.

The piezoelectric response ( $d_{33}$, YE2730, APC, USA) of the poled electrospun nanofibrous membranes was analyzed using a wide-range $d_{33}$ meter. This is a special instrument with a high compliance electrode mechanism ideally suited to thin films and polymers, and can directly measuring the $d_{33}$ values of piezoelectric materials [36]. For the $d_{33}$ characterization, circular specimens were prepared with a diameter of $10 \mathrm{~mm}$ and thickness of $0.15 \mathrm{~mm}$ for each test.

The sound-absorption coefficients of the electrospun PVDF/AgNP nanofibrous membranes were calculated according to the ASTM E1050-08 procedure. A power amplifier provided the signal to a speaker, and the speaker projected the sound waves toward the test sample. Two microphones were placed at a fixed distance apart to measure the sound pressure between the speaker and sample. The sound pressure values were transferred to a data acquisition unit, and the sound-absorption coefficients were calculated using a computer. The sound-absorption coefficient is defined as the amount of sound energy absorbed with respect to the incident energy; a coefficient of 1 indicates $100 \%$ absorption [37]. The two-microphone impedance measurement system consisted of a $99.8 \mathrm{~mm}$-diameter impedance tube (long tube) and a $30.4 \mathrm{~mm}$-diameter impedance tube (short tube). The impedance tubes were used to measure the acoustic absorption and reflection coefficients of the test samples in the $80 \mathrm{~Hz}-6.4 \mathrm{kHz}$ frequency range.

Nanofibrous membranes samples were prepared, and their sound-absorption coefficients were evaluated through impedance-tube measurements. The areal density and porosity of the samples were also measured [38]. The areal densities of the electrospun products with and without AgNPs indicated the same pore size and a similar porosity and surface area, suggesting that all the electrospun samples prepared were comparable.

For the electric output response, the electrospun nanofibrous membranes were sandwiched by flexible $\mathrm{Ni}-\mathrm{Cu}$ conductive electrodes and measured using a source meter unit instrument (SMU, 2400, Keithley, USA). The data were collected and observed using a computer.

\section{Results and discussion \\ 3.1. Morphology of electrospun PVDF/AgNP nanofibrous membranes}

SEM images of the electrospun PVDF nanofibrous membranes prepared without and with AgNPs are shown in Figure 1a and 1b, respectively; the average fiber diameters of these samples were $156 \pm 13$ and $169 \pm 21 \mathrm{~nm}$, respectively. The corresponding TEM images are shown in Figure 1c and 1d. The PVDF nanofiber surface appeared rough under high magnification, and some of the Ag powders were embedded within the nanofibers (Figure 1d). Thus, ultrafine electrospun PVDF nanofibrous membranes featuring a high surface area were successfully prepared with and without AgNPs.

\subsection{Thermal properties of the electrospun PVDF/AgNP nanofibrous membranes}

Figure 2 presents the thermograms of the electrospun PVDF and PVDF/AgNP nanofibrous membranes and the melting and crystallization temperatures measured upon heating and the subsequent cooling of these membranes. Double melting peaks were observed in the first-heating endothermograms of the electrospun PVDF nanofibrous membranes. The results showed that the melting peak at $171^{\circ} \mathrm{C}$ was attributed to the electrospinning-induced melting of the crystalline $\beta$-phase, whereas the peak at $169^{\circ} \mathrm{C}$ was attributed to the melting of the $\alpha$-phase. Conversely, in the first-heating endothermograms of the electrospun PVDF/AgNP, only one melting peak was observed, at $170^{\circ} \mathrm{C}$. The presence of silver led to a higher melting temperature of the $\beta$-phase. Moreover, the cooling exothermograms revealed that the electrospun PVDF/AgNP and electrospun PVDF crystallized at 137 and $131^{\circ} \mathrm{C}$, respectively. The increase in the crystallization temperature, $T_{\mathrm{c}}$, was attributed to silver-induced heterogeneous nucleation, which reveals a strong interaction between silver and the PVDF molecules. 


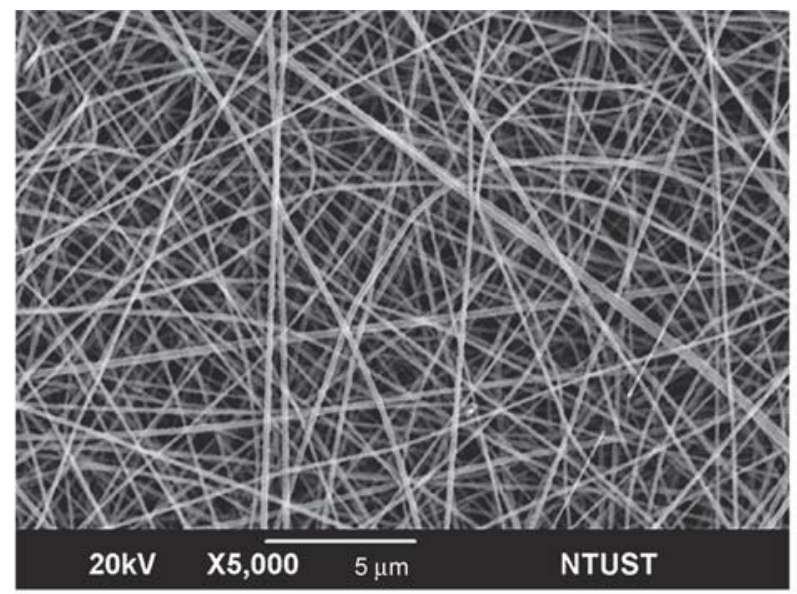

a)

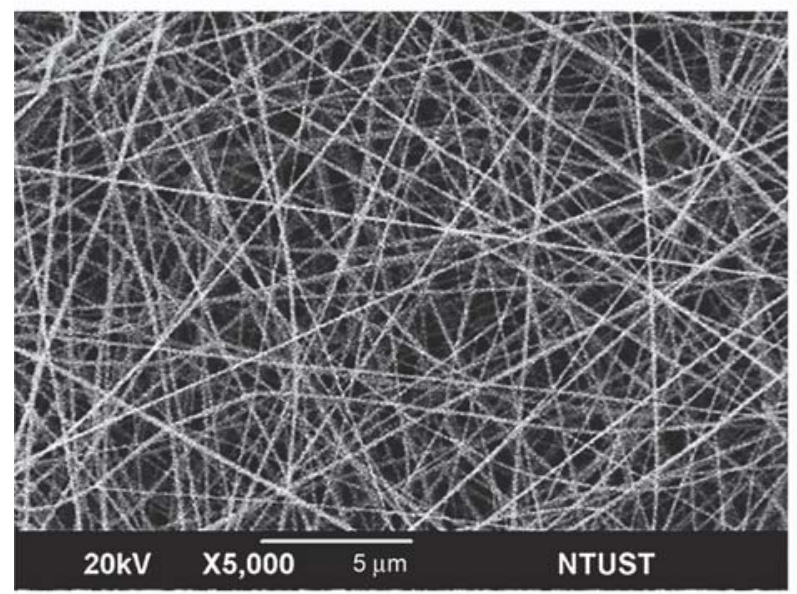

b)

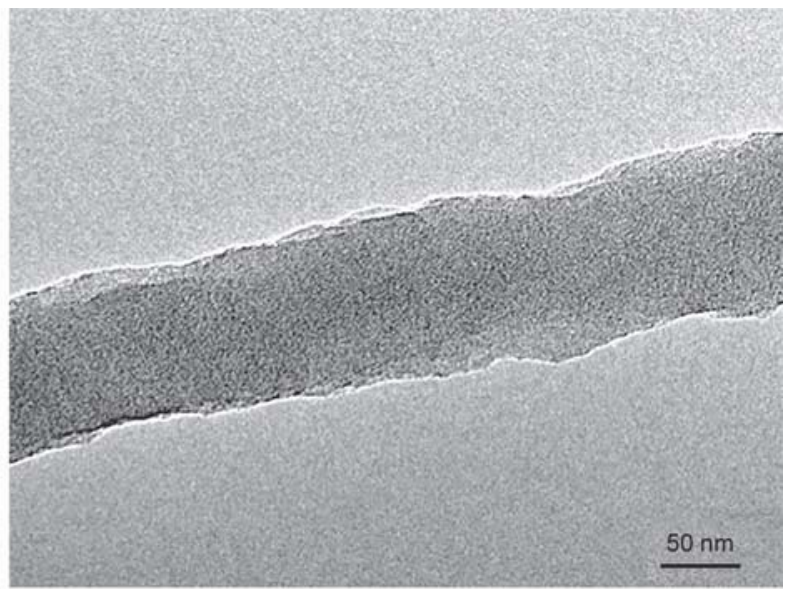

c)

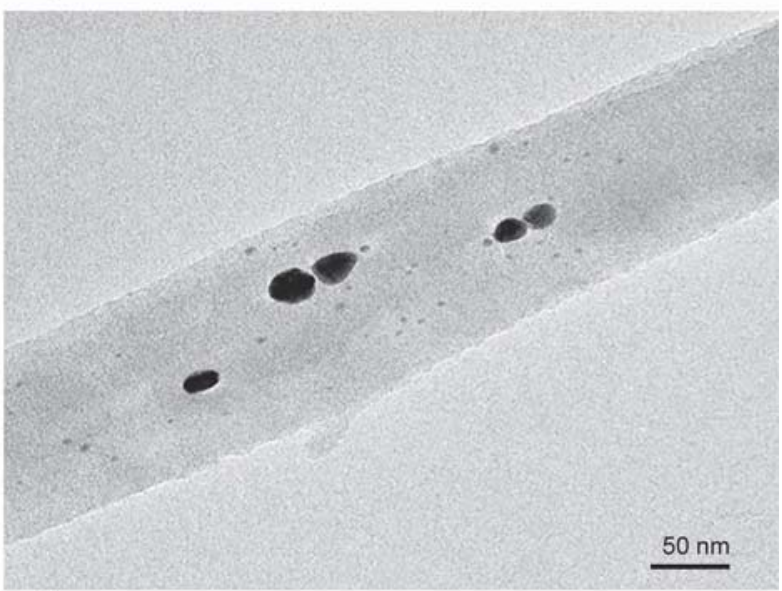

Figure 1. SEM images of the electrospun (a) PVDF and (b) PVDF/AgNP nanofibrous membranes and TEM images of the electrospun (c) PVDF and (d) PVDF/AgNP nanofibers.

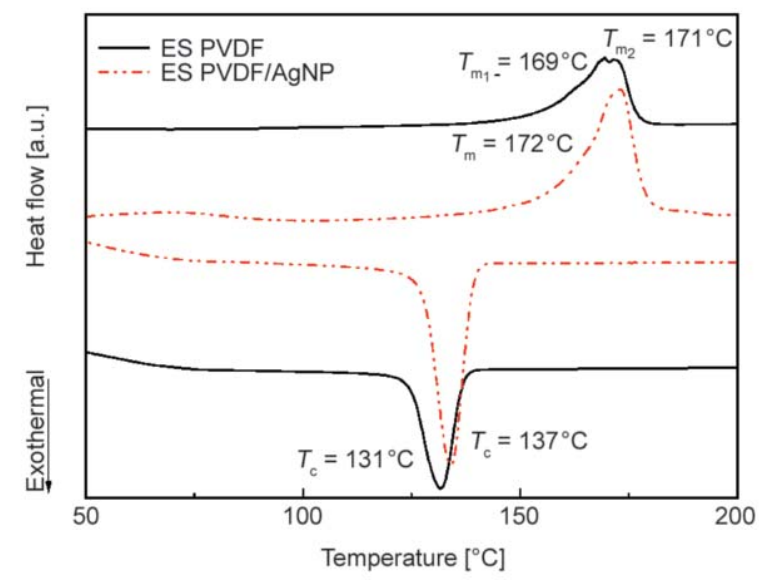

Figure 2. DSC thermogram curves of the electrospun PVDF and PVDF/AgNP nanofibrous membranes.

\subsection{Crystallinity of the electrospun PVDF/AgNP nanofibrous membranes}

FTIR was used to quantify the variation in the crystal phases of PVDF. Figure 3 shows the FTIR spectra for the various PVDF samples. The $\alpha$-phase was resolved at $765,795,855,975$ and $1423 \mathrm{~cm}^{-1}$, and the

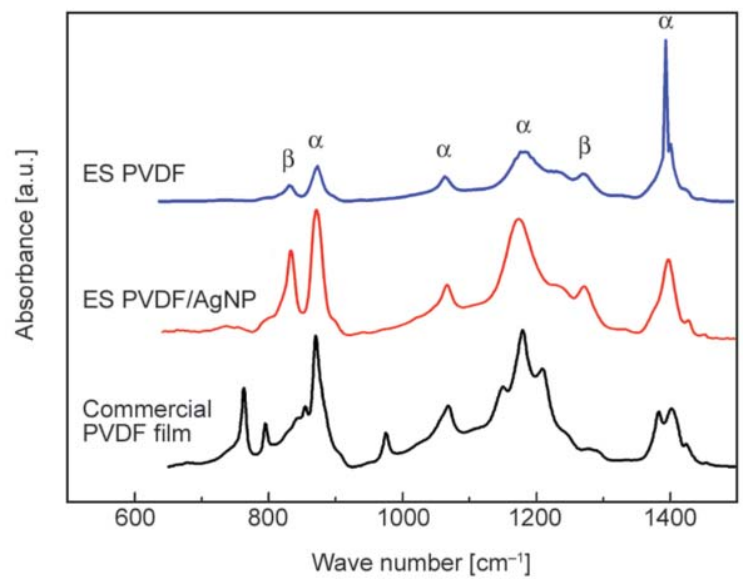

Figure 3. FTIR spectra of the PVDF samples.

$\beta$-phase was resolved at 840 and $1278 \mathrm{~cm}^{-1}$ [39]. As expected, a clear $\beta$-phase was observed in the electrospun PVDF and PVDF/AgNP samples. The relative $\beta$-phase fraction of the PVDF samples, $F(\beta)$, was estimated using FTIR and assumed to follow the Lambert-Beer law (Equation (1)) [39]: 


$$
F(\beta)=\frac{X_{\beta}}{X_{\alpha}+X_{\beta}}=\frac{A_{\beta}}{\left(\frac{K_{\beta}}{K_{\alpha}}\right) A_{\alpha}+A_{\beta}}
$$

where $A_{\alpha}$ and $A_{\beta}$ are the ratios of the incident and absorbed intensity at 765 and $840 \mathrm{~cm}^{-1}$, respectively, and $X_{\alpha}$ and $X_{\beta}$ are the degrees of crystallinity of each phase. $K_{\alpha}$ and $K_{\beta}$ are the absorption coefficients at the corresponding wave number, and are $6.1 \cdot 10^{4}$ and $7.7 \cdot 10^{4} \mathrm{~cm}^{2} \cdot \mathrm{mol}^{-1}$, respectively. The $F(\beta)$ values for the commercial PVDF films [19], electrospun PVDF, and PVDF/AgNP were 14, 83 and 94\%, respectively. Thus, electrospinning and silver addition effectively and significantly induced the formation of the $\beta$ phase.

Furthermore, to determine the optimal parameter for the $\beta$-phase content, different concentrations $(0,0.5$, $1,3,5,10$, and $20 \mathrm{wt} \%$ ) of AgNPs were added to PVDF, as shown in Figure 4. The $\beta$-phase content was the maximum at $1 \mathrm{wt} \%$ of AgNPs content, after which it decreased with an increase in the AgNPs content. This is attributed to the degradation of the local piezoelectric effect of the piezo-conductor hybrid materials when the conductivity is higher than the electrical percolation threshold [30]. Moreover, excess AgNPs may cause more crystal defects, resulting in initial stresses in crystals, and hence, decrease in the degree of crystallinity. Excess nucleating centers also restrict the movement of molecular chains [24]. Thus, the acoustic electric conversion and piezoelectric properties of electrospun PVDF with and without $1 \mathrm{wt} \%$ AgNPs samples were compared.

XPS results for the fine composition of the composite are shown in Figure 5. The binding energy of the electrospun PVDF/AgNP nanofibrous membranes is $367.3 \mathrm{eV}$, which is different from that of AgNP $3 d_{5 / 2}$

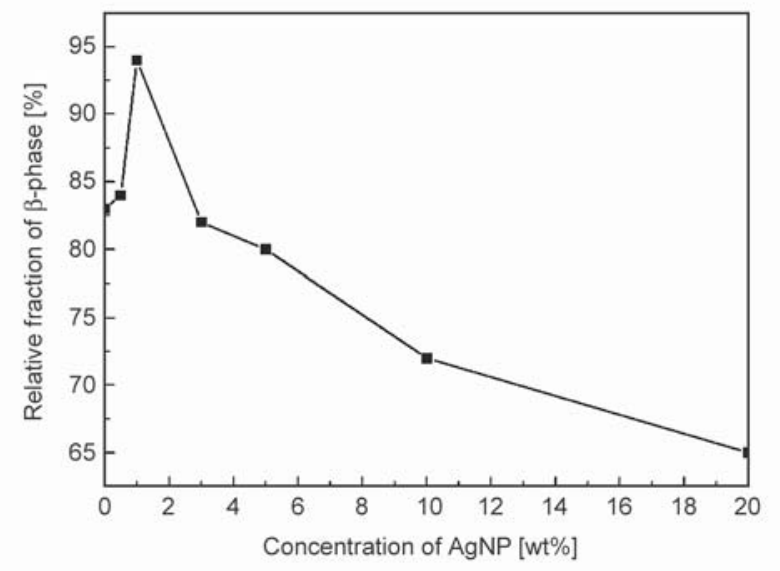

Figure 4. Different concentrations of AgNPs in PVDF samples.

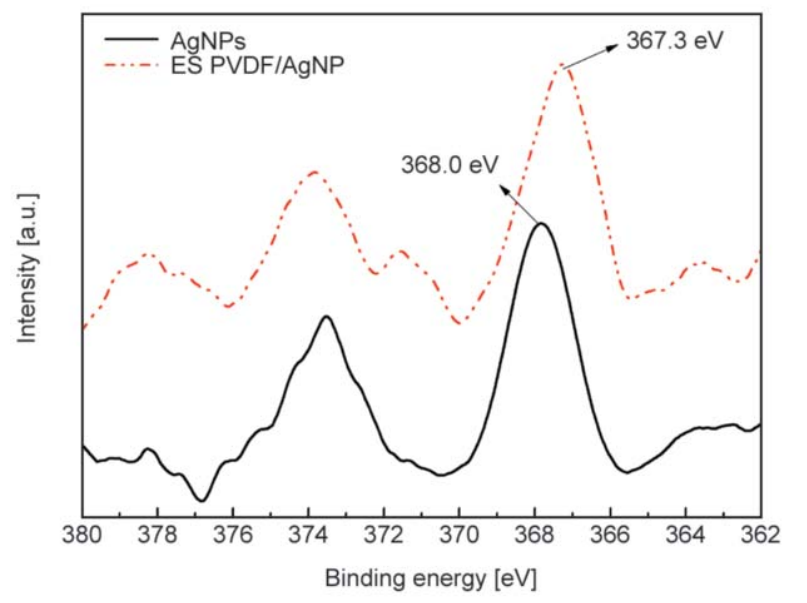

Figure 5. XPS spectra of the AgNPs and the electrospun PVDF/AgNP nanofibrous membrane.

$(368.0 \mathrm{eV})$. Because fluorine is a strong electronegative element and the AgNPs have electron-rich surfaces, the negative shift was attributed to the electrical interactions of the positive silver nanoparticle surfaces with the polymeric $\mathrm{CF}_{2}$ groups of negative charge density [40]. This interaction shows that AgNPs are beneficial for forming the initial $\beta$ crystal nucleus and thus, inducing the polymer chains to align on the surface of the nanoparticles in an extended TTTT conformation, thereby resulting in the formation of the $\beta$-PVDF phase with piezoelectric and ferroelectric properties. Besides, the dielectric constant and the dielectric loss recorded as a function of frequency for electrospun PVDF and PVDF/ AgNPs are shown in Figures S2. The dielectric constant and dielectric loss of $\mathrm{PVDF} / \mathrm{AgNP}$ are higher than PVDF. It is clearly indicated that the introduction of AgNPs in PVDF can enhance polarization from dipole-dipole interaction. Meanwhile, the dielectric loss is considerably higher especially at low frequencies, which is mainly attributed to the conduction of interfacial polarization.

\subsection{Piezoelectricity of the electrospun PVDF/AgNP nanofibrous membranes}

The piezoelectric coefficient $d_{33}$ was measured to examine the relationship between the crystallization and piezoelectricity. Table 1 lists the $F(\beta)$ and $d_{33}$ values. The $d_{33}$ value of the commercial PVDF films was 10.5, and those of the electrospun PVDF and PVDF/AgNP samples were 15.2 and 27.1, respectively. The piezoelectric coefficient increased with the $F(\beta)$ value. However, the results showed that the piezoelectric coefficient of PVDF/AgNP was the highest and that the mobile charge within a conductive phase 
Table 1. $F(\beta)$ values and piezoelectric coefficients of the PVDF samples.

\begin{tabular}{|l|c|c|}
\hline \multicolumn{1}{|c|}{ Sample } & $\begin{array}{c}\boldsymbol{F}(\boldsymbol{\beta}) \\
{[\mathbf{\%}]}\end{array}$ & $\begin{array}{c}\boldsymbol{d}_{\mathbf{3 3}} \\
{[\mathbf{p C} / \mathbf{N}]}\end{array}$ \\
\hline Commercial PVDF film [19] & 14 & 10.5 \\
\hline ES PVDF & 83 & 15.2 \\
\hline ES PVDF/AgNP & 94 & 27.1 \\
\hline
\end{tabular}

(i.e., within the silver phase here) facilitated composite polarization; thus, interfacial polarization occurs in electrically heterogeneous materials. The tendency for polarization can be enhanced by adjusting the permittivity or dielectric constant of the piezoelectric PVDF polymer by incorporating a conductive silver phase within the nanofibers. The increase in the piezoelectricity is explained by the interfacial polarization between the embedded silver phase and polymer matrix.

\subsection{Sound-absorption coefficient of the electrospun PVDF/AgNP nanofibrous membranes}

The sound-absorption coefficients of the electrospun PVDF/AgNP nanofibrous membranes were calculated according to ASTM E1050-08, as shown in Figure 6 . In the low-frequency region $(50-250 \mathrm{~Hz})$, the piezoelectric PVDF products absorbed sound at a lower frequency (approximately $100 \mathrm{~Hz}$ ) than did the nonpiezoelectric acoustic foam. This result was attributed to piezoelectric shunt damping, which reduced the sound transmission of the PVDF membranes at low resonant frequencies [41]. However, the addition of AgNPs improved the piezoelectric properties of the PVDF membrane and further shifted the sound absorption to a lower-frequency region because of the higher $\beta$-phase crystallinity and superior piezoelectric properties. Sound absorption at the

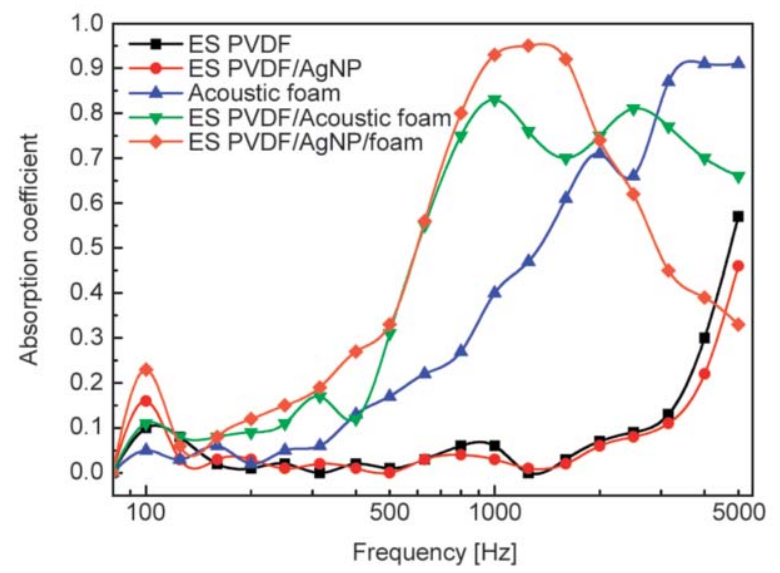

Figure 6. Sound-absorption coefficients of the electrospun PVDF/AgNP and acoustic foam samples. mid-frequency region was mainly converted into thermal energy through a number of mechanisms such as the viscous effect, thermal effect, and material damping. Viscous stresses caused by vibration of the fluid can convert the kinetic energy of the fluid into heat energy. The thermal effect due to the friction at the interface between the fluid and solid also produces heat. Moreover, the impact of an acoustic pressure wave on a solid structure can dissipate energy through a flexible motion of the solid structure owing to the material damping effect. To achieve effective energy exchange between the fluid and the solid, the pressure wave must penetrate deeply enough into the material. Therefore, nanofiber membranes are favorable sound-absorbing materials with many interfaces for the interactions.

For practical applications, the electrospun PVDF/ AgNP nanofibrous membrane was further combined with acoustic foam. The PVDF/AgNP/acoustic foam sample drastically increased the sound-absorbing capability at the mid-frequency region $(315-1250 \mathrm{~Hz})$. This was attributed to the electrospun products providing higher surface areas for contact friction and fine nanofibers for ready vibration: When sound waves propagate within the membranes, the nanofibers readily vibrate and dissipate the sound energy. The sound energy is consumed by the drag between vibrating air particles and the pore surface, which converts the sound energy into thermal energy [37]. Thus, the electrospun PVDF/AgNP/acoustic foam product absorbed more sound than only the acoustic foam did, because of the comparatively higher surface areas of the electrospun products. Piezoelectric PVDF materials absorbed sound in the low-frequency region $(50-250 \mathrm{~Hz})$ because of the improvement in the piezoelectric effect $\left(d_{33}\right)$ achieved by combining electrospinning and the addition of silver. Thus, PVDF acts as an active element (effective at low frequency), whereas the foam acts as a passive element (effective at high frequency). However, the soundabsorption coefficient of the PVDF/AgNP/acoustic foam product decreased in high-frequency region (beyond $2000 \mathrm{~Hz}$ ). The reason is that the electrospun nanofiber membrane is highly reflective to the sound waves in the high-frequency region and results in difficulty movements of sound wave through the materials [42].

Besides, to quantitatively compare sound-absorption performances, the noise reduction coefficient (NRC) was determined. The NRC is the average of 
sound-absorption coefficients at $250,500,1000$, and $2000 \mathrm{~Hz}$ and is often used as an objective measure for practical sound absorption ability at audible frequency [43]. The NRC value for the acoustic foam sample is 0.33 , which is higher than that of the thermally bonded PET nonwovens $(\mathrm{NRC}=0.31$ at a thickness of $15 \mathrm{~mm}$ ) but lower than that of the thicker samples of the thermally bonded PET nonwovens $(\mathrm{NRC}=0.59$, at a thickness of $30 \mathrm{~mm})$ [43]. According to basic acoustic principles, the NRC of samples increases with the frequency and thickness of the nonwovens [43]. The NRC for the PVDF/AgNP/ acoustic foam sample is 0.58 , which is $55 \%$ higher than that obtained for the acoustic foam sample. This value is also higher than that obtained for membranes composed of nonwoven kenaf and electrospun polyamide nanofibers $(\mathrm{NRC}=0.53$ at a thickness of $40 \mathrm{~mm}$ ) [44]. This result implies that the sound absorption properties of conventional sound-absorption materials at audible frequencies can be significantly improved by incorporating a piezoelectric PVDF/ AgNP nanofibrous membrane. Thus, the electrospun $\mathrm{PVDF} / \mathrm{AgNP} /$ acoustic foam has immense practical potential as an efficient sound absorber because of its favorable absorption performance, particularly in the low- and mid-frequency regions.

\subsection{Acoustic electric conversion abilities of the electrospun PVDF/AgNP nanofibrous membranes}

As concluded above, electrospun nanofibrous membranes enhanced the sound energy absorption in the mid-frequency region through the friction and vibration of the internal nanofibers. The piezoelectric electrospun PVDF samples convert sound energy into electric potential, absorbing sound waves in the lowfrequency region. Thus, the acoustic-electric conversion will be frequency dependent. To understand the output voltage of the electrospun PVDF nanofibrous membranes, the PVDF samples were simply sandwiched between the $\mathrm{Ni}-\mathrm{Cu}$ fabric as electrodes and a black Bristol paper was stuck at the bottom as the insulating layer, as shown in Figure S3. The circuit and schematic of testing device is shown in Figure 7, the sound pressure level (SPL) of the actuating sound was set at $\sim 100 \mathrm{~dB}$. Figure 8 shows the output voltage of the PVDF and PVDF/AgNP nanofibrous membranes at different frequencies in the study of acoustic electric conversion characteristics. The output voltages of the PVDF nanofibrous membranes at low, mid- and high frequencies were 49.9, 49.0, and $35.2 \mathrm{mV}$, respectively. The highest electric energy conversion occurs at the low frequency due to the piezoelectric shunt damping of the fiber membrane and increases with increasing $d_{33}$ values. Addition of AgNPs improves the piezoelectric properties of the PVDF membrane and generates a higher output voltage $(59.2,55.8$, and $34.0 \mathrm{mV}$, respectively) at low and mid- frequency range. It is interesting that the piezoelectric fiber membranes generated small voltage signals at the mid- and high frequencies, However, its voltage responses are not sensitive to the piezoelectric coefficient at high-frequency range. The results prove that sound absorption was

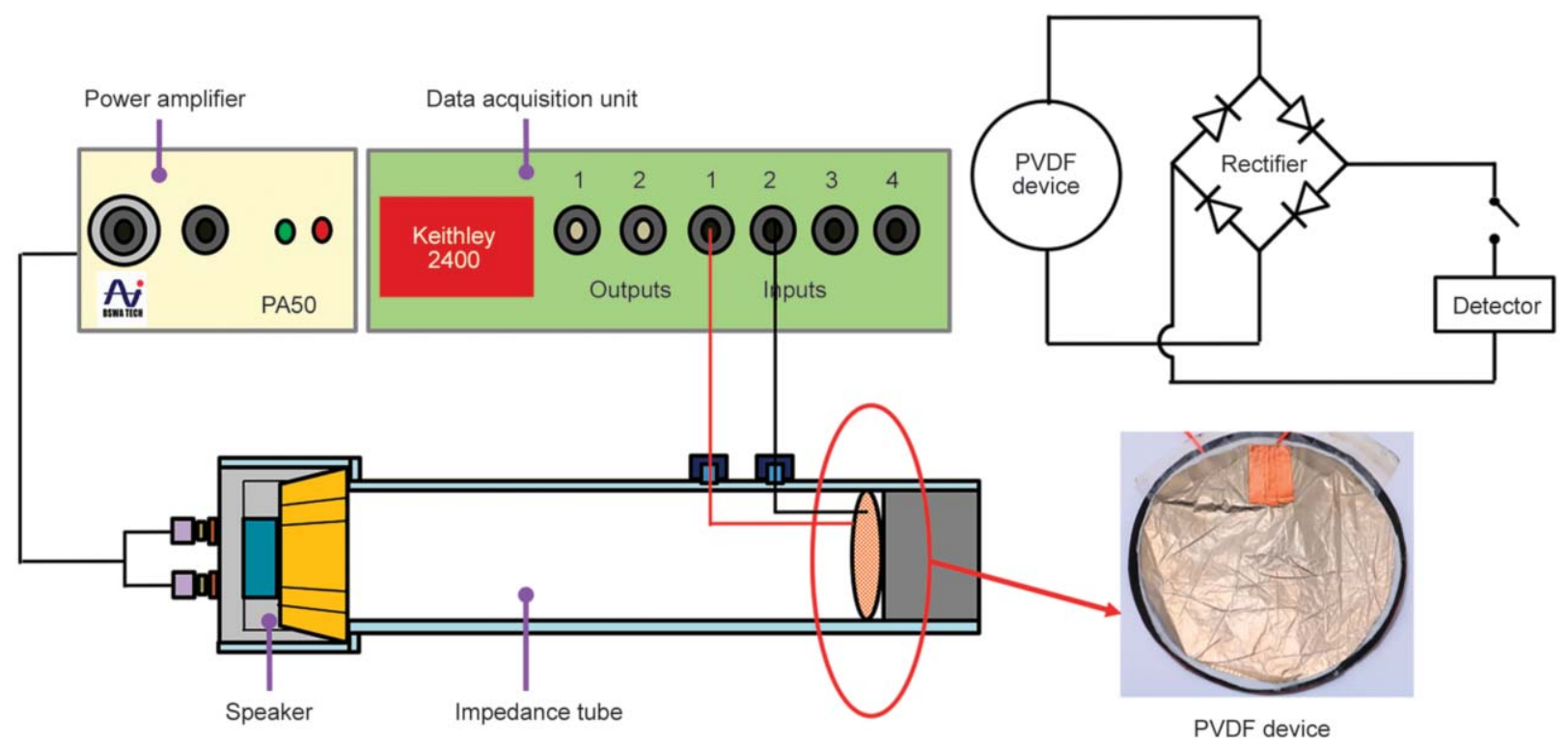

Figure 7. The circuit and schematic of test device for acoustic electric conversion. 

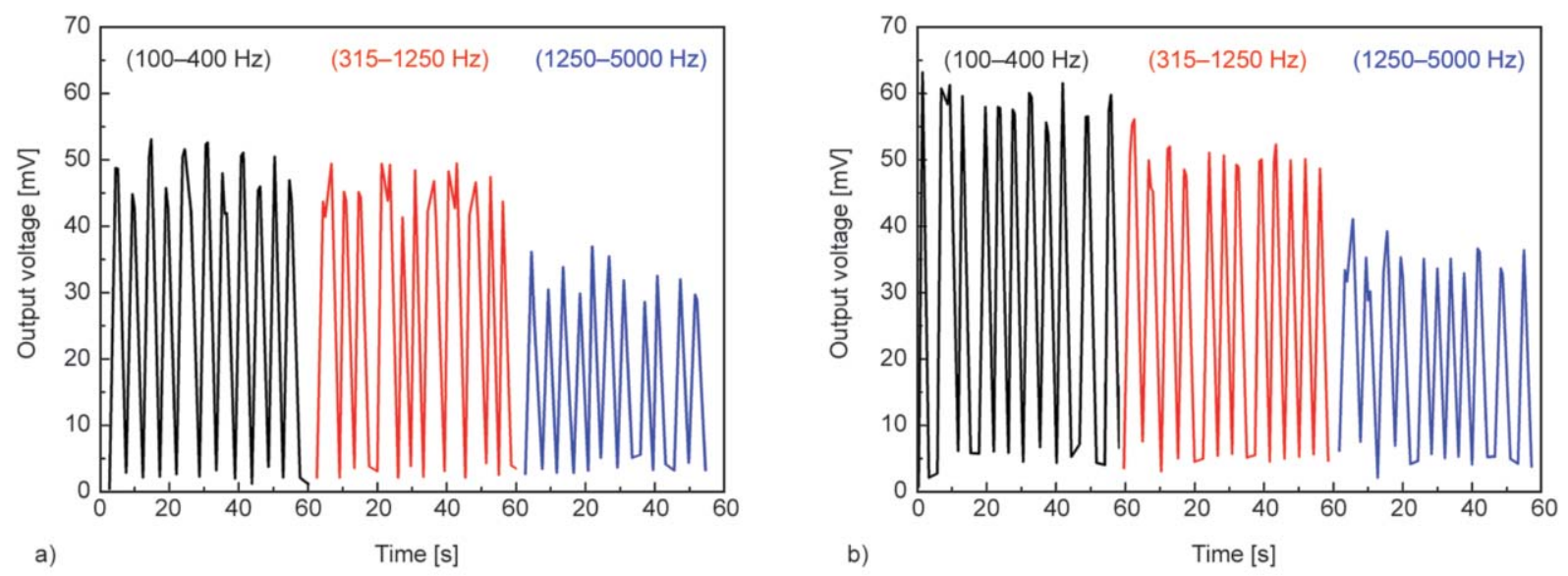

Figure 8. The output voltage of the electrospun a) PVDF and b) PVDF/AgNP at different frequencies.

mainly influenced by viscous, friction, and damping of the nanofibrous membranes at mid- and high frequencies, while the shunt damping and power generation mostly act on the piezoelectric fiber membrane at a low frequency. The conversion of acoustic to electrical energy for ES PVDF/Ag membranes occurs primarily at the low frequency region. Meanwhile, the output electric power of the PVDF/AgNP sample is $7 \cdot 10^{-4} \mathrm{~W}$, which represents a significant increase of approximately $40 \%$ compared with the PVDF sample without AgNPs $\left(5 \cdot 10^{-4} \mathrm{~W}\right)$ at low frequency. Besides, the impedance under different working frequency is also shown in Figure S4. The PVDF was higher than $\mathrm{PVDF} / \mathrm{AgNP}$, indicated that the resistance of PVDF/AgNP is lower and better electrical performance in low frequency. This demonstrates that PVDF/AgNP nanofibrous membranes present excellent piezoelectric properties and acoustic electric conversion characteristics. Therefore, this indicates that the piezoelectric PVDF device was reliable and could be effectively used for sensors or harvester applications, particularly for acoustic-electric conversion purposes.

\section{Conclusions}

In this study, a novel sound energy acquisition which combine sound absorption and power generation based on piezoelectric electrospun PVDF/AgNP nanofibrous membranes was successfully developed. Sound absorption was mainly influenced by viscous, friction, and damping of the nanofibrous membranes at mid- and high Frequency, while the shunt damping and power generation mostly act on the piezoelectric fiber membrane at a low frequency. The conversion of acoustic to electrical energy for ES PVDF/AgNP membranes occurs primarily at the low frequency region. The addition of AgNPs improves the piezoelectric properties of the PVDF membrane and further generates a higher output voltage than PVDF without AgNPs. Meanwhile, the electric power of the PVDF/AgNP is $7 \cdot 10^{-4} \mathrm{~W}$, which represents a significant increase of approximately $40 \%$ compared with that of the PVDF sample without AgNPs $\left(5 \cdot 10^{-4} \mathrm{~W}\right)$ at low frequency. This demonstrates that the PVDF/AgNP nanofibrous membranes present excellent piezoelectric properties and acoustic-electric conversion characteristics. Hence, the piezoelectric PVDF devices can not only reduce sound transmission but also convert sound energy to various other energies. This novel sound energy acquisition device would have immense potential in a variety of sensing and energy harvesting applications and can be advantageous in operating low-power consumer electronic devices and for a green environment.

\section{Supporting information}

The tensile properties of electrospun PVDF samples were shown in Figure S1. PVDF/AgNPs was higher than PVDF.

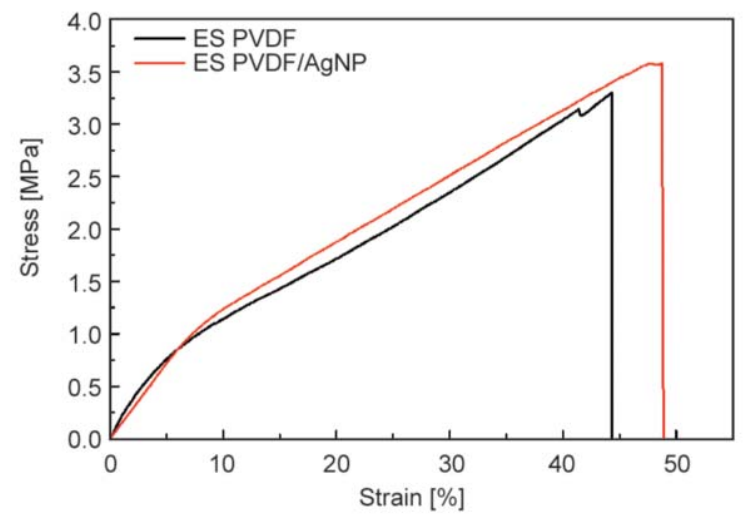

Figure S1. Tensile stress-strain curves of electrospun PVDF and PVDF/AgNP nanofiber membranes. 

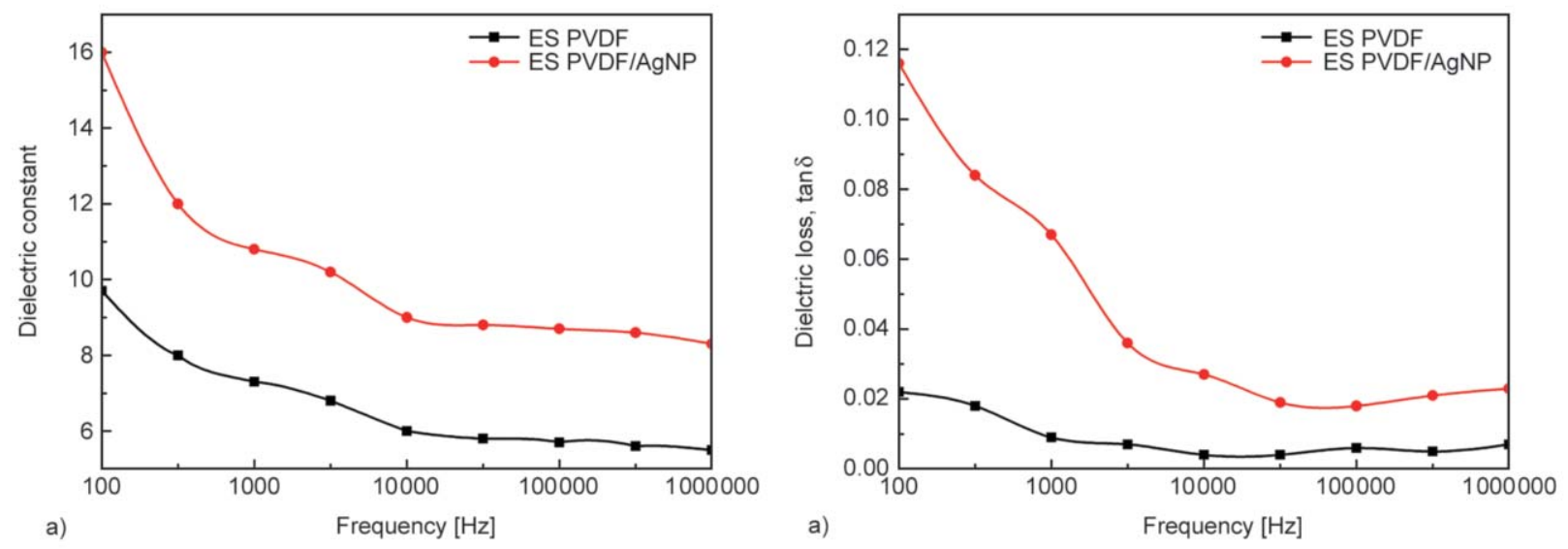

Figure S2. The (a) dielectric constant and (b) dielectric loss of electrospun PVDF and PVDF/AgNP.
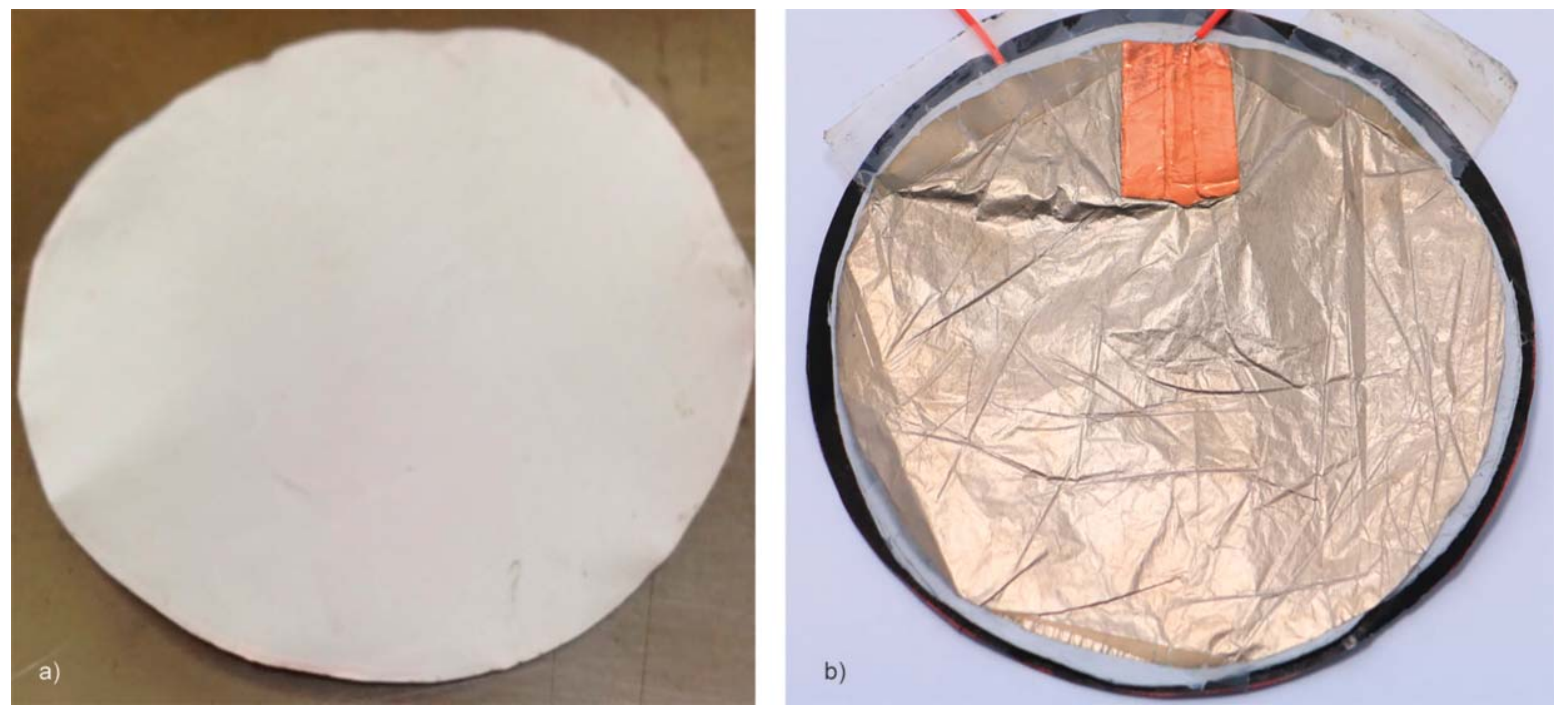

Figure S3. The images of (a) the electrospun PVDF/AgNP nanofibrous membrane and (b) the PVDF/AgNP device.

The room temperature dielectric constant and the dielectric loss recorded as a function of frequency for

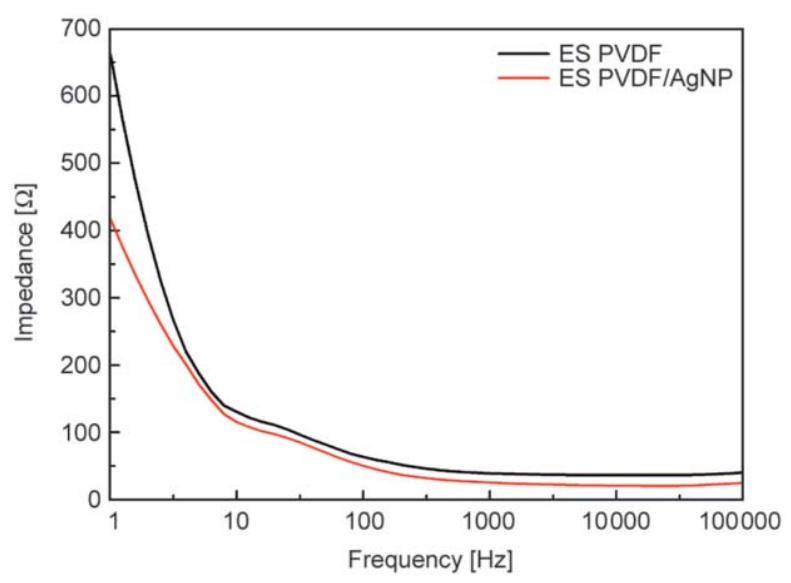

Figure S4. The impedance verse frequency of electrospun PVDF and PVDF/AgNP. electrospun PVDF and PVDF/AgNP are shown in Figure S2. The dielectric constant and dielectric loss of PVDF/AgNP are higher than PVDF.

Figure $\mathrm{S} 3$ is the images of (Figure $\mathrm{S} 3 \mathrm{a}$ ) the electrospun PVDF/AgNP nanofibrous membrane, and (Figure $\mathrm{S} 3 \mathrm{~b}$ ) the $\mathrm{PVDF} / \mathrm{AgNP}$ device that was simply sandwiched between the $\mathrm{Ni}-\mathrm{Cu}$ fabric as electrodes and a black Bristol paper that was stuck at the bottom as the insulating layer.

Figure $\mathrm{S} 4$ shows the result of impedance verse frequency. The PVDF was higher than PVDF/AgNP, indicated that the resistance of $\mathrm{PVDF} / \mathrm{AgNP}$ is lower and better electrical performance in low frequency.

\section{Acknowledgements}

Part of this work was financially supported by the Ministry of Science and Technology of Taiwan, ROC, under the grant number MOST 107-2221-E-011-044. 


\section{References}

[1] Moss S. D., McLeod J. E., Powelesland I. G., Galea S. C.: A bi-axial magnetoelectric vibration energy harvester. Sensors and Actuators A: Physical, 175, 165168 (2012).

https://doi.org/10.1016/j.sna.2011.12.023

[2] Vatansever D., Hadimani R., Shah T., Siores E.: An investigation of energy harvesting from renewable sources with PVDF and PZT. Smart Materials and Structures, 20, 055019/1-055019/7 (2011).

https://doi.org/10.1088/0964-1726/20/5/055019

[3] Gutiérrez A., Dopico N. I., González C., Zazo S., Jiménez-Leube J., Raos I.: Cattle-powered node experience in a heterogeneous network for localization of herds. IEEE Transactions on Industrial Electronics, 60, 3176-3184 (2013).

https://doi.org/10.1109/TIE.2012.2201435

[4] Ouyang Z-W., Chen E-C., Wu T-M.: Enhanced piezoelectric responses and crystalline arrangement of electroactive polyvinylidene fluoride/magnetite nanocomposites. Journal of Applied Polymer Science, 131, 40941/1-40941/8 (2014).

https://doi.org/10.1002/app.40941

[5] Cross L. E.: Ferroelectric materials for electromechanical transducer applications. Materials Chemistry and Physics, 43, 108-115 (1996).

https://doi.org/10.1016/0254-0584(95)01617-4

[6] He F-A., Lin K., Shi D-L., Wu H-J., Huang H-K., Chen J-J., Chen F., Lam K-H.: Preparation of organosilicate/ PVDF composites with enhanced piezoelectricity and pyroelectricity by stretching. Composites Science and Technology, 137, 138-147 (2016).

https://doi.org/10.1016/j.compscitech.2016.10.031

[7] Mahant Y. P., Kondawar S. B., Nandanwar D. V., Koinkar P.: Poly(methyl methacrylate) reinforced poly (vinylidene fluoride) composites electrospun nanofibrous polymer electrolytes as potential separator for lithium ion batteries. Materials for Renewable and Sustainable Energy, 7, 5/1-5/9 (2018).

https://doi.org/10.1007/s40243-018-0115-y

[8] Ahn Y., Lim J., Hong S. M., Lee J., Ha J., Choi H. J., Seo Y.: Enhanced piezoelectric properties of electrospun poly(vinylidene fluoride)/multiwalled carbon nanotube composites due to high $\beta$-phase formation in poly (vinylidene fluoride). The Journal of Physical Chemistry C, 117, 11791-11799 (2013).

https://doi.org/10.1021/jp4011026

[9] Kim N. K., Lin R. J. T., Fakirov S., Aw K., Bhattacharyya D.: Nanofibrillar poly(vinylidene fluoride): Preparation and functional properties. International Journal of Polymeric Materials and Polymeric Biomaterials, 63, 23-32 (2014).

https://doi.org/10.1080/00914037.2013.769244

[10] Kang D. H., Kang H. W.: Advanced electrospinning using circle electrodes for freestanding PVDF nanofiber film fabrication. Applied Surface Science, 455, 251257 (2018).

https://doi.org/10.1016/j.apsusc.2018.05.211
[11] Merlini C., Pegoretti A., Araujo T. M., Ramoa S. D. A. S., Schreiner W. H., de Oliveira Barra G. M.: Electrospinning of doped and undoped-polyaniline/poly(vinylidene fluoride) blends. Synthetic Metals, 213, 34-41 (2016). https://doi.org/10.1016/j.synthmet.2015.12.024

[12] Zeng Z., Liu M., Xu H., Liao Y., Duan F., Zhou L-M., Jin H., Zhang Z., Su Z.: Ultra-broadband frequency responsive sensor based on lightweight and flexible carbon nanostructured polymeric nanocomposites. Carbon, 121, 490-501 (2017).

https://doi.org/10.1016/j.carbon.2017.06.011

[13] Ouyang Z-W., Chen E-C., Wu T-M.: Enhanced piezoelectric and mechanical properties of electroactive polyvinylidene fluoride/iron oxide composites. Materials Chemistry and Physics, 149, 172-178 (2015). https://doi.org/10.1016/j.matchemphys.2014.10.003

[14] Patra S. N., Lin R. J. T., Bhattacharyya D.: Regression analysis of manufacturing electrospun nonwoven nanotextiles. Journal of Materials Science, 45, 3938-3946 (2010).

https://doi.org/10.1007/s10853-010-4459-7

[15] Zhang B., Kang F., Tarascon J-M., Kim J-K.: Recent advances in electrospun carbon nanofibers and their application in electrochemical energy storage. Progress in Materials Science, 76, 319-380 (2016).

https://doi.org/10.1016/j.pmatsci.2015.08.002

[16] Duan G., Greiner A.: Air-blowing-assisted coaxial electrospinning toward high productivity of core/sheath and hollow fibers. Macromolecular Materials and Engineering, 304, 1800669/1-1800669/5 (2019).

https://doi.org/10.1002/mame.201800669

[17] Wu C-M., Chou M-H., Zeng W-Y.: Piezoelectric response of aligned electrospun polyvinylidene fluoride/ carbon nanotube nanofibrous membranes. Nanomaterials, 8, 420/1-420/13 (2018).

https://doi.org/10.3390/nano8060420

[18] Wu C. M., Chou M. H.: Sound absorption of electrospun polyvinylidene fluoride/graphene membranes. European Polymer Journal, 82, 35-45 (2016).

https://doi.org/10.1016/j.eurpolymj.2016.07.001

[19] Wu C. M., Chou M. H.: Polymorphism, piezoelectricity and sound absorption of electrospun PVDF membranes with and without carbon nanotubes. Composites Science and Technology, 12, 127-133 (2016). https://doi.org/10.1016/j.compscitech.2016.03.001

[20] Neppalli R., Wanjale S., Birajdar M., Causin V.: The effect of clay and of electrospinning on the polymorphism, structure and morphology of poly(vinylidene fluoride). European Polymer Journal, 49, 90-99 (2013). https://doi.org/10.1016/j.eurpolymj.2012.09.023

[21] Wang J. C., Chen P., Chen L., Wang K., Deng H., Chen F., Zhang Q., Fu Q.: Preparation and properties of poly (vinylidene fluoride) nanocomposites blended with graphene oxide coated silica hybrids. Express Polymer Letters, 6, 299-307 (2012). https://doi.org/10.3144/expresspolymlett.2012.33 
[22] Jiang S., Chen Y., Duan G., Mei C., Greiner A., Agarwal S.: Electrospun nanofiber reinforced composites: A review. Polymer Chemistry, 9, 2685-2720 (2018). https://doi.org/10.1039/c8py00378e

[23] Hu L., Kim H., Lee J-Y., Peumans P., Cui Y.: Scalable coating and properties of transparent, flexible, silver nanowire electrodes. ACS Nano, 4, 2955-2963 (2010). https://doi.org/10.1021/nn1005232

[24] Wu L., Huang G., Hu N., Fu S., Qiu J., Wang Z., Ying J., Chen Z., Lia W., Tang S.: Improvement of the piezoelectric properties of PVDF-HFP using AgNWs. RSC Advances, 4, 35896-35903 (2014). https://doi.org/10.1039/C4RA03382E

[25] Li B., Xu C., Zheng J., Xu C.: Sensitivity of pressure sensors enhanced by doping silver nanowires. Sensors, 14, 9889-9899 (2014). https://doi.org/10.3390/s140609889

[26] Issa A. A., Al-Maadeed M. A., Luyt S. A., Ponnamma D., Hassan M. K.: Physico-mechanical, dielectric, and piezoelectric properties of PVDF electrospun mats containing silver nanoparticles. Journal of Carbon Research, 3, 30/1-30/16 (2017).

https://doi.org/10.3390/c3040030

[27] Gwon J. G., Kim S. K., Kim J. H.: Sound absorption behavior of flexible polyurethane foams with distinct cellular structures. Materials and Design, 89, 448-454 (2016). https://doi.org/10.1016/j.matdes.2015.10.017

[28] Xue B., Li R., Deng J., Zhang J.: Sound absorption properties of microporous poly(vinyl formal) foams prepared by a two-step acetalization method. Industrial and Engineering Chemistry Research, 55, 3982-3989 (2016). https://doi.org/10.1021/acs.iecr.6b00127

[29] Kundu A., Berry A.: Active sound control with smart foams using piezoelectric sensoriactuator. Journal of Intelligent Material Systems and Structures, 22, $1771-$ 1787 (2011). https://doi.org/10.1177/1045389X11409079

[30] Rahimabady M., Statharas E. C., Yao K., Mirshekarloo M. S., Chen S., Francis E. H. T.: Hybrid local piezoelectric and conductive functions for high performance airborne sound absorption. Applied Physics Letters, 111, 241601/1-241601/5 (2017). https://doi.org/10.1063/1.5010743

[31] Hori M., Aoki T., Ohira Y., Yano S.: New type of mechanical damping composites composed of piezoelectric ceramics, carbon black and epoxy resin. Composites Part A: Applied Science and Manufacturing, 32, 287-290 (2001). https://doi.org/10.1016/S1359-835X(00)00141-X

[32] Lang C., Fang J., Shao H., Ding X., Lin T.: High-sensitivity acoustic sensors from nanofibre webs. Nature Communications, 7, 11108/1-11108/7 (2016). https://doi.org/10.1038/ncomms11108
[33] Meyers F. N., Loh K. J., Dodds J. S., Baltazar A.: Active sensing and damage detection using piezoelectric zinc oxide-based nanocomposites. Nanotechnology, 24, 185501/1-185501/10 (2013). https://doi.org/10.1088/0957-4484/24/18/185501

[34] Yu X., Rajamani R., Stelson K. A., Cui T.: Carbon nanotube-based transparent thin film acoustic actuators and sensors. Sensors and Actuators A: Physical, 132, 626631 (2006).

https://doi.org/10.1016/j.sna.2006.02.045

[35] Ghafari E., Lu N.: Self-polarized electrospun polyvinylidene fluoride (PVDF) nanofiber for sensing applications. Composites Part B: Engineering, 160, 1-9 (2019). https://doi.org/10.1016/j.compositesb.2018.10.011

[36] Han J., Li D., Zhao C., Wang X., Li J., Wu X.: Highly sensitive impact sensor based on PVDF-TrFE/nano-ZnO composite thin film. Sensors, 19, 830/1-830/13 (2019). https://doi.org/10.3390/s19040830

[37] Ma Z., Kotaki M., Yong T., He W., Ramakrishna S.: Surface engineering of electrospun polyethylene terephthalate (PET) nanofibers towards development of a new material for blood vessel engineering. Biomaterials, 26, 2527-2536 (2005). https://doi.org/10.1016/j.biomaterials.2004.07.026

[38] El Achaby M., Arrakhiz F. Z., Vaudreuil S., Essassi E. M., Qaiss A., Bousmina M.: Preparation and characterization of melt-blended graphene nanosheets-poly(vinylidene fluoride) nanocomposites with enhanced properties. Journal of Applied Polymer Science, 127, 46974707 (2013).

https://doi.org/10.1002/app.38081

[39] Salimi A., Yousefi A. A.: Analysis method: FTIR studies of $\beta$-phase crystal formation in stretched PVDF films. Polymer Testing, 22, 699-704 (2003). https://doi.org/10.1016/S0142-9418(03)00003-5

[40] Chou H. L., Wu C. M., Lin F. D., Rick J.: Interactions between silver nanoparticles and polyvinyl alcohol nanofibers. AIP Advances, 4, 087111/1-087111/10 (2014). https://doi.org/10.1063/1.4890290

[41] Duan X. H., Wang H. Q., Li Z. B., Zhu L. K., Chen R., Kong D. Y., Zhao Z.: Sound absorption of a flexible micro-perforated panel absorber based on PVDF piezoelectric film. Applied Acoustics, 88, 84-89 (2015). https://doi.org/10.1016/j.apacoust.2014.08.009

[42] Seddeq H. S.: Factors influencing acoustic performance of sound absorptive materials. Australian Journal of Basic and Applied Sciences, 3, 4610-4617 (2009).

[43] Lee Y. E., Joo C. W.: Sound absorption properties of thermally bonded nonwovens based on composing fibers and production parameters. Journal of Applied Polymer Science, 92, 2295-2302 (2004). https://doi.org/10.1002/app.20143

[44] Iannace G.: Acoustic properties of nanofibers. Noise and Vibration Worldwide, 45, 29-33 (2014). https://doi.org/10.1260/0957-4565.45.10.29 